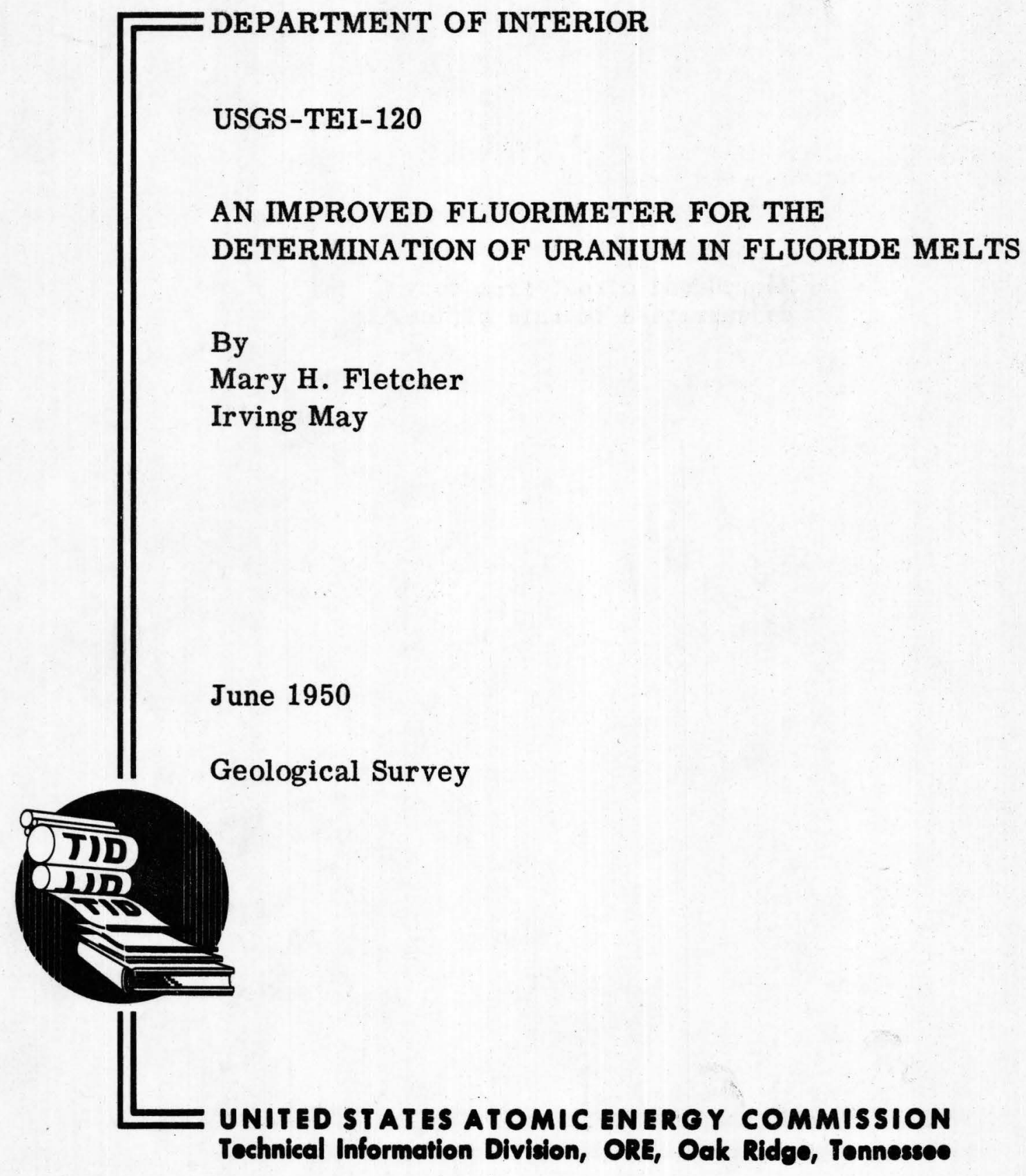


Reproduced direct from copy as submitted to this office. 
Argonne National Laboratory

Armed Forces Special Weapons Project

Atomic Energy Commission, Washington

Battelle Memorial Institute

Brookhaven National Laboratory

Carbide and Carbon Chemicals Division, UCCC, K-25 Plant

Carbide and Carbon Chemicals Division, UCCC, Y-12 Plant

Columbia University (Failla)

Dow Chemical Company

General Electric Company, Richland

Idaho Operations Office

Iowa state College

Kellex Corporation

Knolls Atomic Power Laboratory

Los Alamos

Mallinckrodt Chemical Works

Massachusetts Institute of Technology (Gaudin)

Massachusetts Institute of Technology (Kaufmann)

Mound Laboratory

National Advisory Committee for Aeronautics

National Bureau of Standards

Naval Medical Research Institute

Naval Radiological Defense Laboratory

NEPA Project

New Brunswick Laboratory

New York Operations Office

North American Aviation, Inc.

Oak Ridge National Laboratory

Patent Branch, Washington

Sylvania Electric Products, Inc.

Technical Information Service, ORE, Oak Ridge

UCLA Medical Research Laboratory (Warren)

University of California Radiation Laboratory

University of Rochester

Western Reserve University (Friedell)

Westinghouse Flectric Corporation

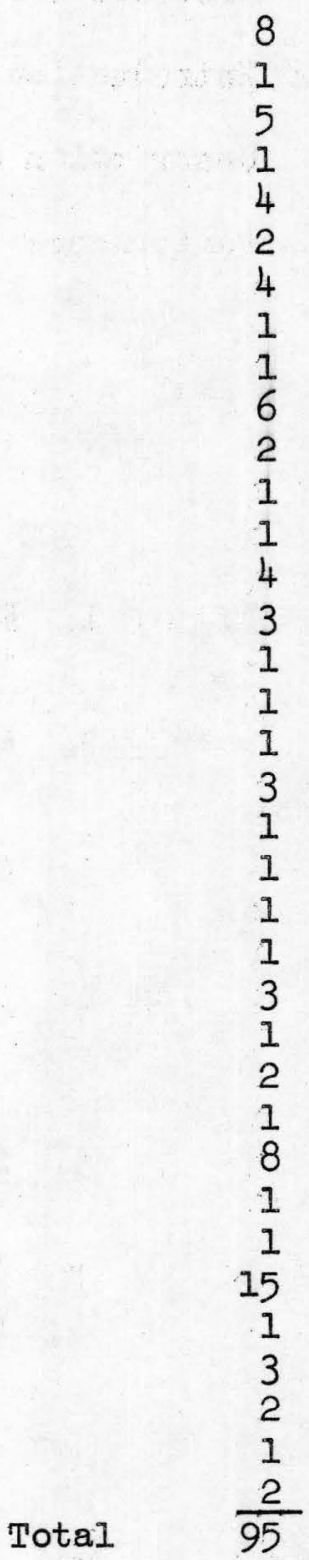


CONTENTS

Page

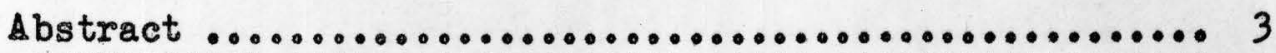

Introduction $\ldots \ldots \ldots \ldots \ldots \ldots \ldots \ldots \ldots \ldots \ldots \ldots \ldots \ldots \ldots \ldots \ldots \ldots$

Description of the instrument $\ldots \ldots \ldots \ldots \ldots \ldots \ldots \ldots \ldots \ldots$

Performance of the instrument $\ldots \ldots \ldots \ldots \ldots \ldots \ldots \ldots \ldots \ldots \ldots \ldots$

\section{ILLUSTRA TIONS}

Page

Figure 1. Expanded details of the modified

fluorimeter ......................... 11

2. Rear view assembly of the

modified fluorimeter ................ I2

3. Complete assembly of the modified

fluorimeter

4. Standardization curves for $3.0 \mathrm{gg}$

discs prepared by fusion in

30-ml crucibles ....................... 14

5. Standardization curves for $1.5 \mathrm{gg}$

discs prepared by fusion in

crucible lids ........................ 35

6. Calibration curve for routine work ........ 16 
AN IMPROVED FLUORIMETER FOR THE DETERMINATION OF URANIUM IN FLUORIDE MELTS

by

Wary H. Fletcher and Irving May

\begin{abstract}
ABSTRA.CT
The Model $R$ fluorimeter has been modified to increase its stability and sensitivity. The new instrument* is about ten times as sensitive as the original fluorimeter, but it can also be employed conveniently at a sensitivity level comparable to or less than that of the Model $\mathrm{R}$ fluorimeter.

The modified fluorimeter described here was developed in the latter half of 1948 and has since been in constant use in this laboratory for routine analysis. Although it is now being superseded to a large extent by a more recently developed transmission fluorimeter, the modified fluorimeter is a very useftul tool for many types of analysis. (Fletcher, M. H., May, Irving, and Slavin, Morris, A transmission fluorimeter for use in the fluorimetric method of analysis for uranium: Trace Elements Investigations Report 104, August 1949.)
\end{abstract}


INTRODUCTION

The Oak Ridge Model $R$ fluorimeter $I /$ was in constant use

1/Coleman, C.F., C-4.381.4, 1946.

at the Geological Survey for six months and proved to be very useful. When it became increasingly apparent that a more sensitive and stable instrument would better meet the particular. needs of this laboratory, an improved fluorimeter for use in the analysis of low-grade samples was constructed. The new instrument possesses the following advantages over the Model $R$ fluorimeter:

1. Greater sensitivity

2. Increased electrical stability

3. Greater flexibility, by which a wider range of fluorescence can be measured

In the Model $R$ fluorimeter a Hanovia Utility lamp is fastened directly to the instrument, and there is no provision for cooling the lamp, although it is characteristic of highpressure mercury lamps that their intensity is, among other things, a function of their ambient temperature. As a result, when the instrument is used for 30 to 40 minutes after a 10minute warmup period, there is a decrease of approximately 30 percent in the instrumental sensitivity because of increased lamp temperature. It is, therefore, frequently necessary to 
turn the lamp off, allow it to $\operatorname{cool}_{3}$ and then restart it in order to maintain a usable range of sensitivity. This is not only a time-consuming process, but it is a poor practice inasmach as such lamps undergo the greatest wear during the starting period. Furthermore, the high operating temperature of the unventilated Utility lamp, as it is used in the Model $R$ instrument, raises the temperature of the phototube and causes a constant drift of the zero of about four or five divisions after each reading. Such a change introduces some uncertainty in the readings. It also necessitates resetting the zero point after each reading which is both time consuming and a source of annoyance to the operator.

In the first model of the new fluorimeter, the minimum changes consistent with our requirements were made, and several parts of the Model $\mathrm{R}$ instrument were incorporated in the modified fluorimeter. The substitution of the more intense General Electric E-H 4 or C-H 4 lamp for the Hanovia Utility lamp in the new instrument increases its sensitivity yet allows the continued use of the Photovolt Electronic Photometer (Model 512). The lamp is supported on a stand and is ventilated by a Bon-Air ventilating fan (obtainable at photographic stores). After a 30-minute initial warmup the operating temperature remains constant, and the lamp can be left on all day if desired; furthermore, the phototube remains $\operatorname{cool}_{3}$ and most of the drift is eliminated. 
A feature of the new fluorimeter is a convenient sensitivity control beyond the 25-percent change in sensitivity possible with the Photovolt unit. As the lamp is not mounted directly on the instrument, the distance between the lamp and sample may be changed readily. Extra sensitivity control results from the variation in intensity of illumination according to the inverse square law as the distance between the sample and lamp is changed. For instance, with the E-H 4 lamp 12 inches above the sample ( $8 \frac{1}{2}$ inches above the primary filter) and the Photovolt unit set for lowest sensitivity, the standard glass supplied with the Model $R$ fluorimeter will give a reading of 1000. This is about twice the usable sensitivity of the Model $\mathrm{R}$ fluorimeter. When the lamp is moved to within 6 inches of the sample ( 3 inches from the primary filter), the sensitivity is increased to eight times the sensitivity of the Model R instrument. For still greater sensitivity a $\mathrm{C}-\mathrm{H} 4$ lamp is used about $1 \frac{1}{2}$ inches above the primary filter; this arrangement gives a sensitivity about ten times that of the Model $R$ fluorimeter.

\section{DESCRIPTION OF THE INSTRUMENT}

The modified instrument is built on the base of the Model $R$ fluorimeter and retains the sample slide, the Photovolt measuring unit, and the secondary filters of the old instrument. The housing for the filters and shutter is made from 26-gauge sheet brass painted a flat black (figs, 1, 2, and 3)。A No. 6 Packard shutter, obtainable from photographic shops, with a $1 \frac{1}{2}$-inch-diameter opening 
18 attached directly above the secondary filters. The top plate of the shutter is replaced with a bakelite cover having a $1 \frac{1}{4}-i n c h-$ diameter hole in the center. One end of a brass collar is held in this hole by a friction fit, and the other end is threaded to screw directly into the search unit. All joints are secure and light tight. The secondary filters are the yellow and green filters (Corning No. 3486 and No. 9780, moulded thickness) from

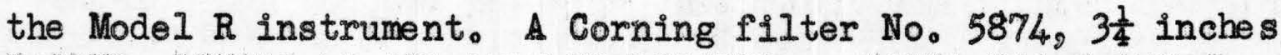
square, serves as the primary filter. A sheet metal shield provided with air vents is placed around the instrument, and the enclosure is ventilated with a Bon-Air ventilating fan.

\section{PERFORMANCE OF THE INSTRUMENT}

The instrument equipped with an $\mathrm{E}-\mathrm{H} 4$ lamp was first tested with the glass standard provided with the Model R fluorimeter. After a 10-minute warmup period the distance of the lamp was adjusted to give a reading of 1000 . After 20 minutes the reading had dropped to 900 . Readings of 900 were then obtained at inter = vals over a period of five hours. The initial drop from 1000 to 900 indicates the need for at least 30 -minute warmup period to obtain equilibrium.

The performance of the instrument was then tested with standard samples. The se were of two kinds: first, a series of 12 standards containing from 0 to 3 micrograms of uranium prepared by fusion in 30-ml platinum crucibles with 3 grams of 
fusion mixture;2/ second, a series of 8 standards containing from

2/ 9 percent $\mathrm{NaF}$ in $\mathrm{Na}_{2} \mathrm{CO}_{3}, \mathrm{~K}_{2} \mathrm{CO}_{3}(1+1$ by weight). The pellets were removed from the crucibles before the measurements were made.

0 to 0.15 microgram of uranium prepared by fusion in platinum lids with 1.5 grams of the same flux. Each of these two sets of standards was read in the instrument with the lamp $8 \frac{1}{2}$ and 3 inches above the primary filter. As many as 10 to 20 discs could be read without resetting the dark current. The curves obtained in these experiments as well as a comparison curve obtained with the Model $R$ fluorimeter are given in figures 4 and 5.

The modified fluorimeter has now been in constant use for almost two years and has proved to be very satisfactory. However, future models may incorporate such changes as a different lamp support to give better and easier lamp adjustment, a built-in slide for cutting the intensity of the exciting light, a Sola constant-voltage transformer designed for the operation of the H 4 lamps (catalog No. 30825), and possibly a different base and sample holder. The Photovolt Corporation has installed two additional ranges in some of our instruments. A $2 \mathrm{X}$ and a $5 \mathrm{X}$ scale have been added to the original "Hi" and "Lo" scales. The se new ranges increase the precision for those readings just beyond the range of the "Hi" scale. 
Many of the samples analyzed in this laboratory contain less than 0.02 percent of uranium, and few contain more than 0.04 percent. These determinations are usually made by the method described by Grimaldi and Levine.3/ An aliquot which contains $3.75 \mathrm{mg}$ of sample

3/ Grimaldi, F。 So, and Levine, H., The rapid fluorimetric determination of uranium in low-grade ores; a preliminary reports Trace Elements Investigations Rept。 47, April 1948.

is used for the preparation of the phosphors. At the present time one of the instruments has been adapted specifically to the convenient handling of the se samples.

For this purpose a $\mathrm{C}-\mathrm{H} 4$ lamp $1 \frac{1}{2}$ inches above the primary filter is the source of exciting light, and only the "Lo" scale of the Photorolt instrument is used. Standard curves are drawn to give direct readings in percent of uranium (fig. 6). Curve A (0 to 0.019 percent uranium) was obtained when the glass standard (a Corning polished glass filter, No. 3384,2 inches square, masked down to a 0.8 -inch-diameter circle) read 80 divisions: in this curve one scale division is equivalent to 0.00022 percent uranium. For curve $B$ ( 0 to 0.066 percent uranium) the intensity of the exciting light was cut down by interposing 2 nichrome gauze squares between the lamp and the instrument. With this arrangement the glass standard read 25 divisions, and one scale division was equivalent to 0.00068 percent uranium. Once the curves are prepared, it is necessary only to set the instrument to give the proper reading with the glass standard. 
Occasional checks of the curve are made with uranium standards, particularly when new batch of flux is used.

Acknowledgment.--The authors are indebted to Melvin E. Hanes of the Geological Survey who made the drawings and graphs for this paper. 


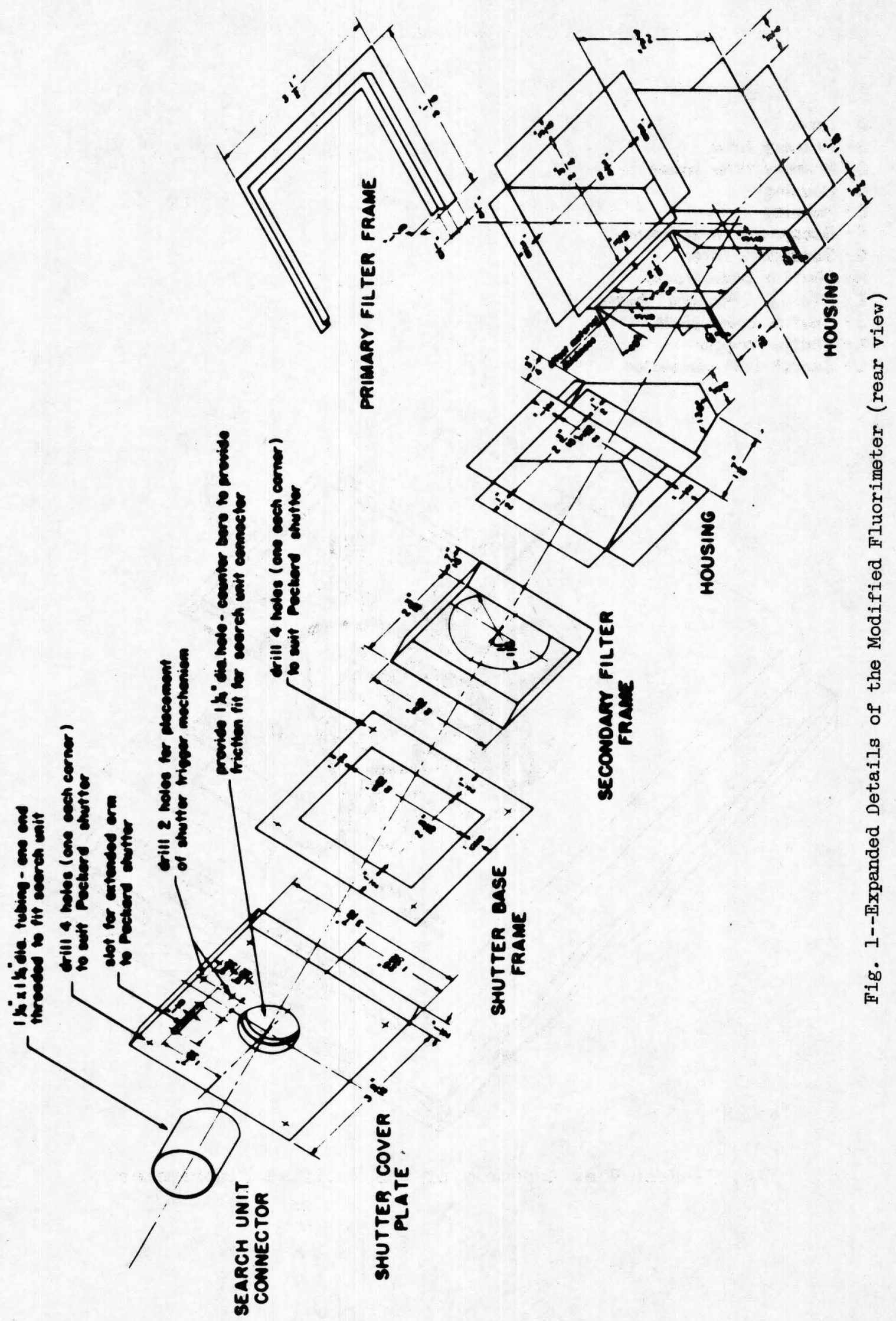




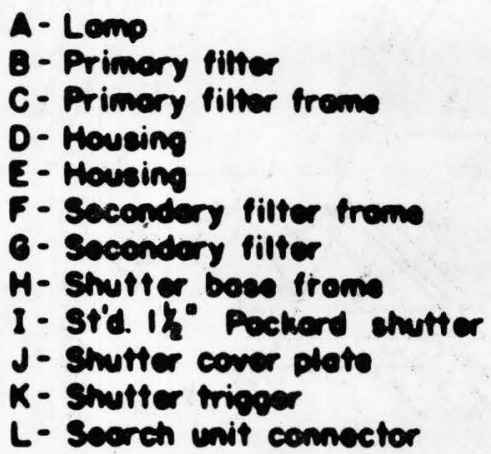

A - Lemp

8- Primery pilter

trem

0- Housing

- Horsin

F- Secendery filter from

O- Secendery filter

H- Snutter boes from

1- stid. 1/ Pesticen churter

- sintier coner ploto

L- Secreh unit connector

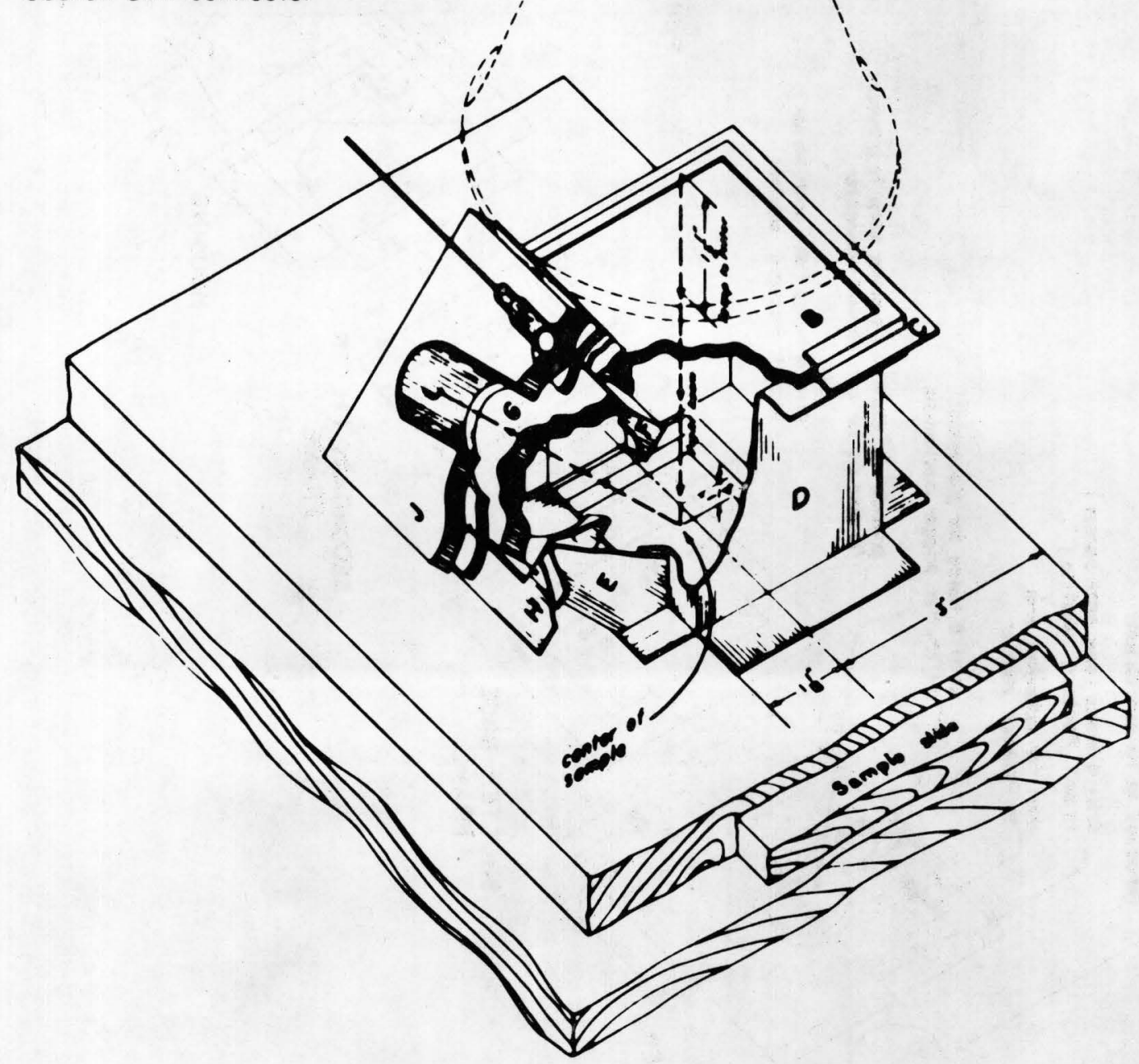

Fig. 2--Rear View Assembly of the Modified Fluorimeter 


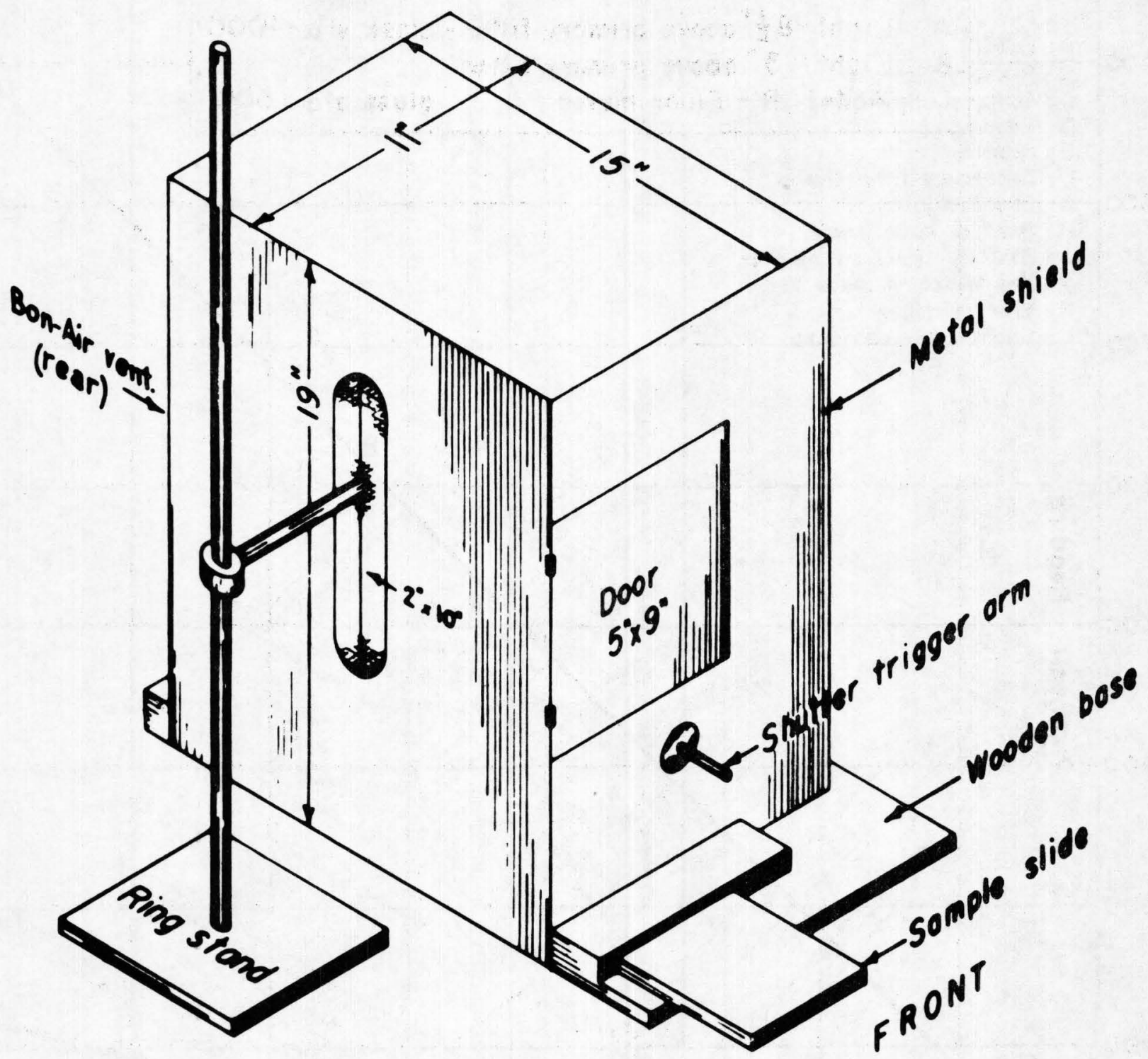

Fig. 3--Complete Assembly of the Modified Fluorimeter (front view) 


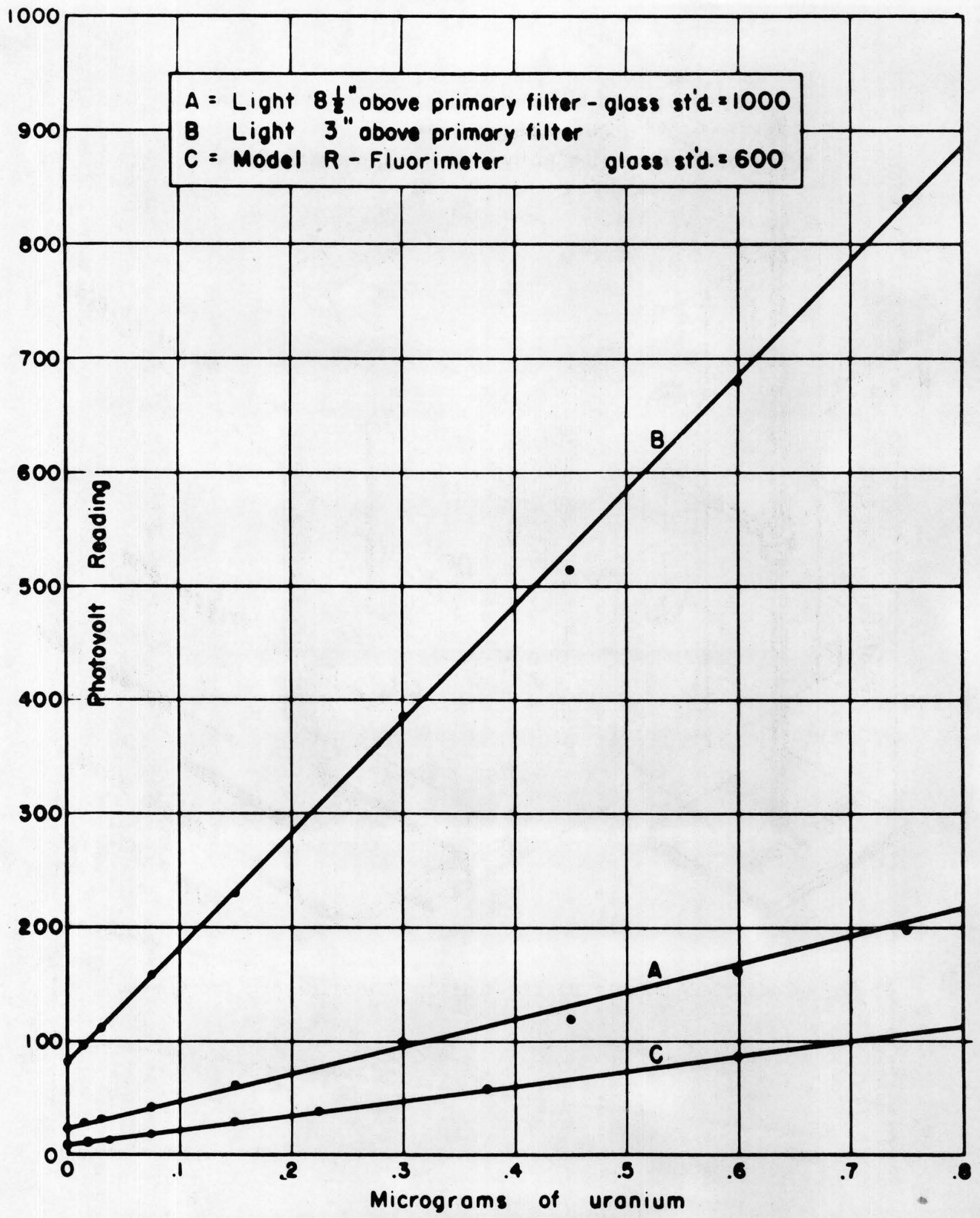

Fig. 4--Standardization curves for $3.0-\mathrm{g}$ discs prepared by fusion in 30ml crucibles. 


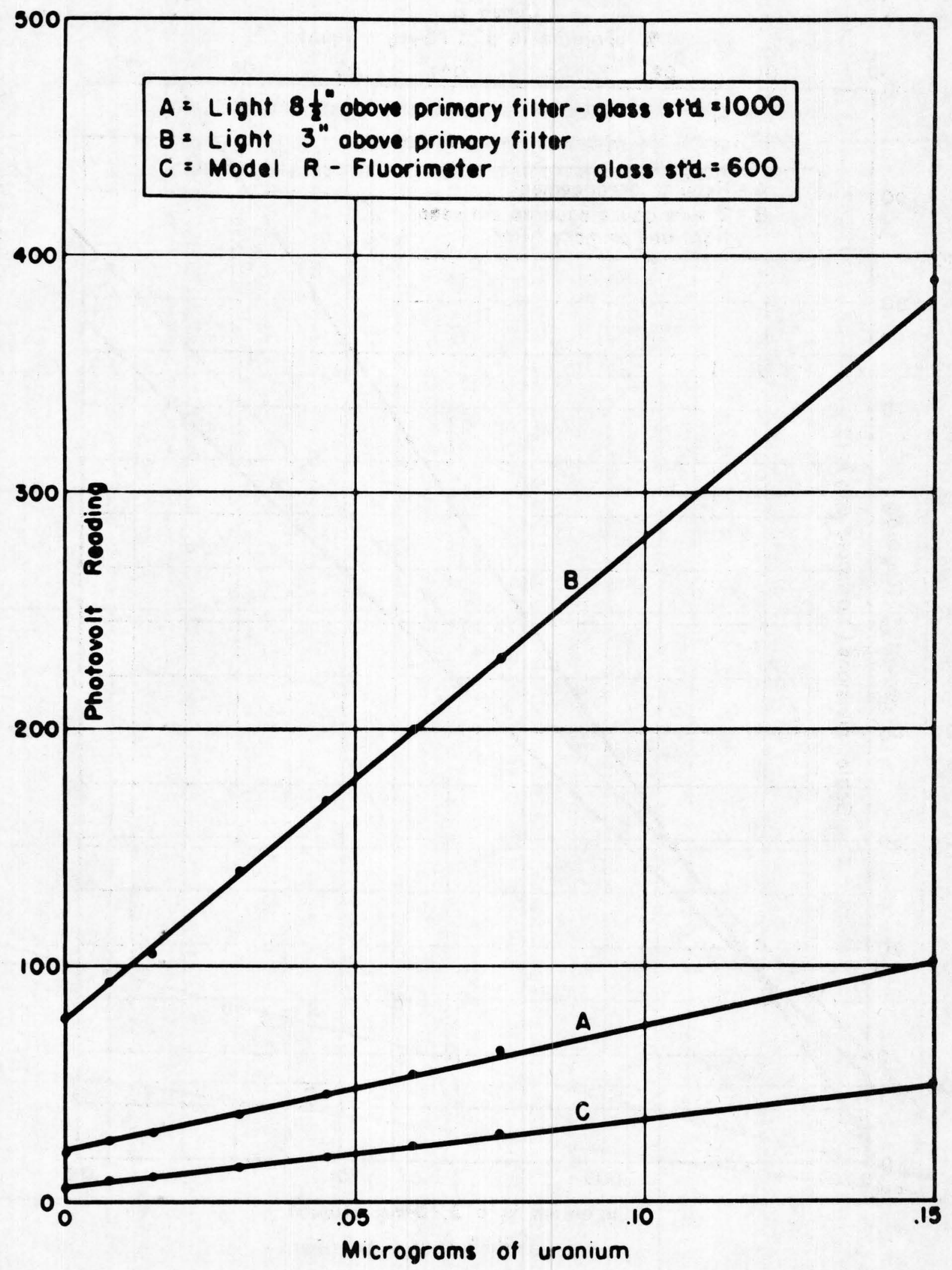

Fig. 5--Standardization curves for 1.5-g discs prepared by fusion in crucible lids. 
Curve $B$

\% uronium in o $3.75-\mathrm{mg}$ oliquot

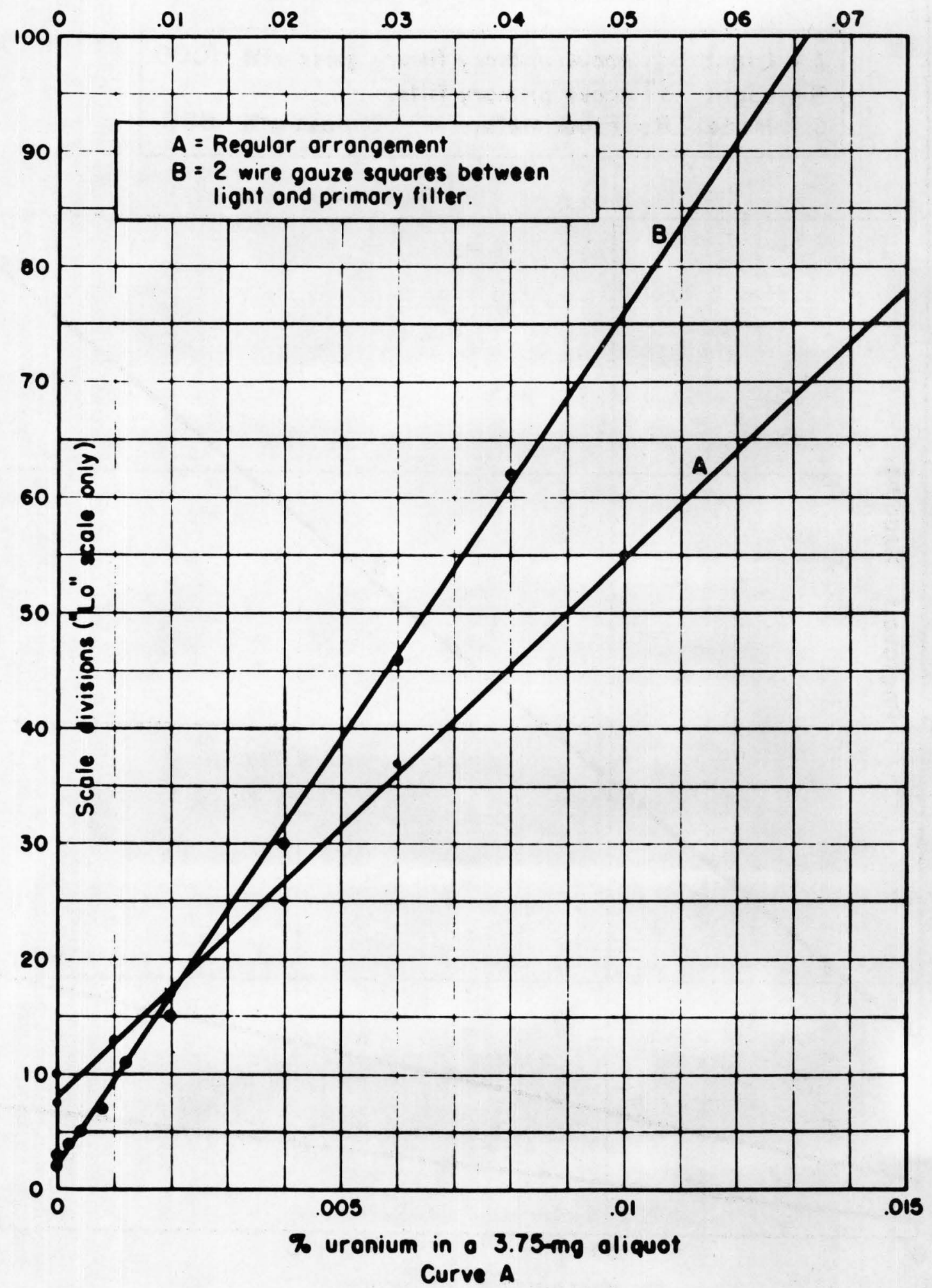

Fig. 6--Calibration curve for routine work. 